\title{
Variabilidad epigenética en la producción de embriones in vitro de dos bovinos clonados por bipartición embrionaria
}

\section{Epigenetic variability in the production of in vitro embryos from two boves cloned by embryo bipartition}

\author{
Miguel Ángel Arista Ruiz ; Nilton Luis Murga Valderrama²
}

\section{RESUMEN}

En la presente investigación se determinó y comparó variabilidad epigenética en la producción de embriones in vitro de dos bovinos homocigotos de la raza Aberdeen Angus. Los ovocitos se obtuvieron de matadero y fueron madurados y fecundados por el método convencional. Posteriormente se cultivaron por 7 días en gotas de $90 \mu \mathrm{l}$ de medio fluido oviductual sintético (SOF) cubiertos con aceite mineral. A los 7 días de cultivo se evaluó la producción total de embriones (blastocistos iniciales (Bi), blastocistos (Bl) y blastocistos expandidos $(\mathrm{Bx})$ ), presentando mayor porcentaje el ejemplar JTRM 27.86\% a comparación del ejemplar VTRM 23.86\% (p<0.05) y encontrando diferencia estadística significativa.

Palabras clave: epigenética, embriones, bovinos, clonación.

\begin{abstract}
In the present investigation, epigenetic variability in the production of in vitro embryos of two homozygous cattle of the Aberdeen Angus breed was determined and compared. The oocytes were obtained from slaughterhouse and were matured and fertilized by the conventional method. Subsequently, they were grown for 7 days in $90 \mu 1$ drops of synthetic oviductual fluid medium (SOF) covered with mineral oil. After 7 days of culture, the total production of embryos (initial blastocysts $(\mathrm{Bi})$, blastocysts $(\mathrm{Bl})$ and expanded blastocysts $(\mathrm{Bx})$ ) was evaluated, with a higher percentage of the JTRM specimen $27.86 \%$ compared to the VTRM specimen $23.86 \%(\mathrm{p}<0.05)$ and finding significant statistical difference.
\end{abstract}

Keywords: epigenetics, embryos, cattle, cloning.

${ }^{1}$ Bachiller en Ingeniería Zootecnista de la Universidad Nacional Toribio Rodríguez de Mendoza de Amazonas.

${ }^{2}$ Médico Veterinario. Investigador del Instituto de Ganadería y Biotecnología de la Universidad Nacional Toribio Rodríguez de Mendoza de Amazonas. E-mail: nmurga.fizab@untrm.edu.pe 


\section{INTRODUCCIÓN}

En las ciencias médicas los gemelos homocigotos constituyen un grupo idóneo para abordar el estudio de las variabilidades epigenéticas, etc. La epigenética estudia las variaciones hereditarias que ocurren sin que cambie la secuencia del ADN, es decir, de los cambios en la función de los genes que no afectan la secuencia del ADN, si no por modificaciones que tienen lugar principalmente en las citosinas de éste y en las histonas de la cromatina. Se ha determinado que las modificaciones epigenéticas son mucho más frecuentes que aquellas que modifican la secuencia del $\mathrm{ADN}$, por lo que constituyen uno de los fundamentos de la diversidad biológica. (Gonzales, Díaz y Díaz-Anzaldúa, 2008)

El conocimiento de la fertilidad o de la capacidad fecundante de cada toro es el principal objetivo en la producción de semen bovino (Quintero, Mayorga y Cardona, 2017).

La predicción de la capacidad fecundante del esperma es técnica y económicamente muy importante, en especial cuando se trabaja con reproductores que están en centros de inseminación. Los investigadores en el mundo han desarrollado varias pruebas relacionadas con la calidad de semen y su morfología (Barth, 1992), motilidad espermática (Kjaestad et al., 1993; Holt et al., 1997), componentes bioquímicos del semen (Hirao, 1975), presencia de cromosoma intacto (Correa et al., 1997), integridad de membrana plasmática (Perez et al., 1997), concentración de espermatozoides seleccionados por swim up (Zhang et al., 1998), reacción acrosómica (Januskauskas et al., 2000), etc. La producción de embriones in vitro se ha desarrollado y utilizado para la producción de crías de alto valor genético de diferentes especies (Palmer et al., 1991). Esta técnica implica la maduración in vitro de ovocitos (MIV), fecundación in vitro de ovocitos (FIV) y el cultivo in vitro de ovocitos fecundados (CIV) hasta el estadio de blastocisto. A través de los años, muchos estudios, realizados principalmente en bovinos, han demostrado que el donante del semen influye en gran medida el resultado fecundación y cultivo de embriones (Shi et al., 1990; Shamsuddin y Larsson, 1993).

El mayor éxito del procedimiento de fecundación in vitro se demuestra al producir individuos vivos, utilizando tanto ovocitos madurados in vivo o in vitro, aunque la eficiencia de estos procesos de FIV disminuye respecto a la fecundación natural, de igual modo que cuando se utilizan ovocitos madurados in vivo frente a los madurados in vitro (Lorenzo et al., 1994).

\section{MATERIAL Y MÉTODOS}

1Bachiller en Ingeniería Zootecnista de la Universidad Nacional Toribio Rodríguez de Mendoza de Amazonas.

2Médico Veterinario. Investigador del Instituto de Ganadería y Biotecnología de la Universidad Nacional Toribio Rodríguez de Mendoza de A m a zon a s. E - $\mathrm{m}$ a i 1 : nmurga.fizab@untrm.edu.peLugar de estudio La investigación se realizó en el Laboratorio de Biotecnología Animal, Reproducción y Mejoramiento Genético de la Universidad Nacional Toribio Rodríguez de Mendoza de Amazonas (UNTRM).

Materiales

Tabla 1: Materiales y equipos usados para la producción de embriones in vitro

\begin{tabular}{lll}
\hline \multicolumn{1}{c}{ Materiales } & Material biológico y reactivos & \multicolumn{1}{c}{ Equipos } \\
\hline Termo de transporte de ovarios & Ovarios recolectados de camal & Baño maría \\
Tijera & Solución salina al $0.9 \%$ & Estereoscopio \\
Termómetro & Medio de manipulación & Microscopio \\
Set de Tips de $10 \mu 1,20 \mu 1,100 \mu 1,200 \mu 1$ y $1000 \mu 1$ & Medio de maduración & Cabina de bioseguridad \\
Guantes quirúrgicos & & \\
Set de Micropipetas de $10 \mu 1,20 \mu 1,100 \mu 1,200 \mu 1$ y $1000 \mu 1$ & Medio de fecundación & Incubadora \\
Papel toalla & & estacionaria \\
Botas de jebe & Medio de cultivo \\
Overol & Semen bovino & Platina térmica \\
Vaso bicker & Percoll al $45 \%$ & Centrífuga \\
Jeringa de $10 \mathrm{ml}$ & Percoll al $90 \%$ & Balón de gas CO2 \\
Aguja hipodérmica $18 \mathrm{G}$ & Alcohol al $70 \%$ & \\
Tubos falcon de $15 \mathrm{ml}$ & Aceite mineral & \\
Pipetas Pasteur & Agua ultrapura & \\
Placas de $35,70 \mathrm{y} 100 \mathrm{~mm}$ & Agua destilada & \\
Tubos eppendorf & & \\
Portaobjetos y cubreobjetos & & \\
\hline
\end{tabular}




\section{Selección de los animales donantes de óvulos}

Las vacas Brown swiss cruzadas a donar los ovarios se seleccionaron del camal municipal de la ciudad de Chachapoyas, previo al beneficiado, se realizó una evaluación ante mortem como el estado sanitario, edad del animal por el número de sus incisivos, seleccionando a los que se encuentren entre 3 y 5 años indicativo que ya se encuentren en edad reproductiva y que por lo menos hayan tenido un parto (4 - 8 dientes) (Casas et al., 2001).

Obtención de los ovarios y aspiración del complejo ovocito-cúmulos (COCs)

Para la recolección de los ovarios se tuvo en cuenta que se encuentren todas las estructuras ováricas como folículo primario, folículo secundario, cuerpo hemorrágico, cuerpo lúteo, etc. Los óvulos se recuperaron de los folículos ováricos entre los $2 \mathrm{~mm}$ y $7 \mathrm{~mm}$ a través de la técnica de aspiración folicular con una jeringa hipodérmica de $10 \mathrm{ml}$ y una aguja $18 \mathrm{G}$. Finalmente se realizó la búsqueda y categorización (calidad A y B) (De Loos et al., 1992 y Sato et al., 1990) en un estereoscopio mediante la observación.

\section{Maduración in vitro de los complejos ovocito- cúmulos (COCs)}

Después de la recuperación y clasificación, los COCs de calidad A y B (viables) fueron lavados dos veces en medio de manipulación. Se colocaron 25 óvulos por microgota de $90 \mu \mathrm{l}$ con medio de maduración, cubiertas con $4 \mathrm{ml}$ de aceite mineral, en placas FIV de $35 \mathrm{~mm}$, que previamente fue equilibrada como mínimo por 2 horas en condiciones de 5\% CO2, 38,5 ${ }^{\circ} \mathrm{C}$ y humedad sobre $90 \%$ en la incubadora.

Fertilización in vitro de los complejos ovocitocúmulos (COCs)

Para la fertilización se usó semen criopreservado de dos bovinos gemelos homocigotos clonados por bipartición embrionaria de raza Aberdeen Angus; JTRM y VRTM que fueron clonados por bipartición embrionaria.

Es importante afirmar también que al momento de la colecta, el semen de estos toros; al evaluar las características seminales como volumen, $\mathrm{pH}$, motilidad masal, motilidad individual, concentración y morfología, no presentaban diferencias estadísticas significativas entre ambos ejemplares.

Después de las 24 horas de maduración en promedio, los ovocitos fueron fecundados por 18 horas, se adicionó $10 \mu \mathrm{l}$ de espermatozoide por microgota, seleccionados por el método de Percoll y diluido en igual proporción con medio de fecundación.

\section{Cultivo in vitro de embriones}

Los embriones resultantes de la FIV, aproximadamente 18 horas post inseminación, se desnudaron los ovocitos casi completamente por pipeteo en las mismas gotas de medio de fecundación y se lavaron en medio de cultivo antes de transferirlos a microgotas de medio de cultivo CIV de $90 \mu \mathrm{l}$, cubiertas de $4 \mathrm{ml}$ de aceite mineral, donde los embriones continuaron su división celular a 8,16 y 32 células hasta el día 7 de cultivo y llegar al estadío de blastocistos.

\section{Diseño de la investigación}

Se utilizó el diseño pre experimental con post pruebas en dos grupos y con muestras diferentes. Se realizaron en total 24 repeticiones por cada toro.

\section{RESULTADOS}

\section{Producción total de embriones}

La producción total de embriones es la sumatoria de los embriones que llegaron hasta los estadíos de blastocisto inicial, blastocisto y blastocisto expandido, la figura 1 muestra el número de embriones por repetición de los dos toros. El porcentaje total de embriones para el toro JTRM fue de $27.86 \%$ a, mientras que para el toro VTRM fue de $23.86 \%$ b de un total de 244 óvulos fecundados.

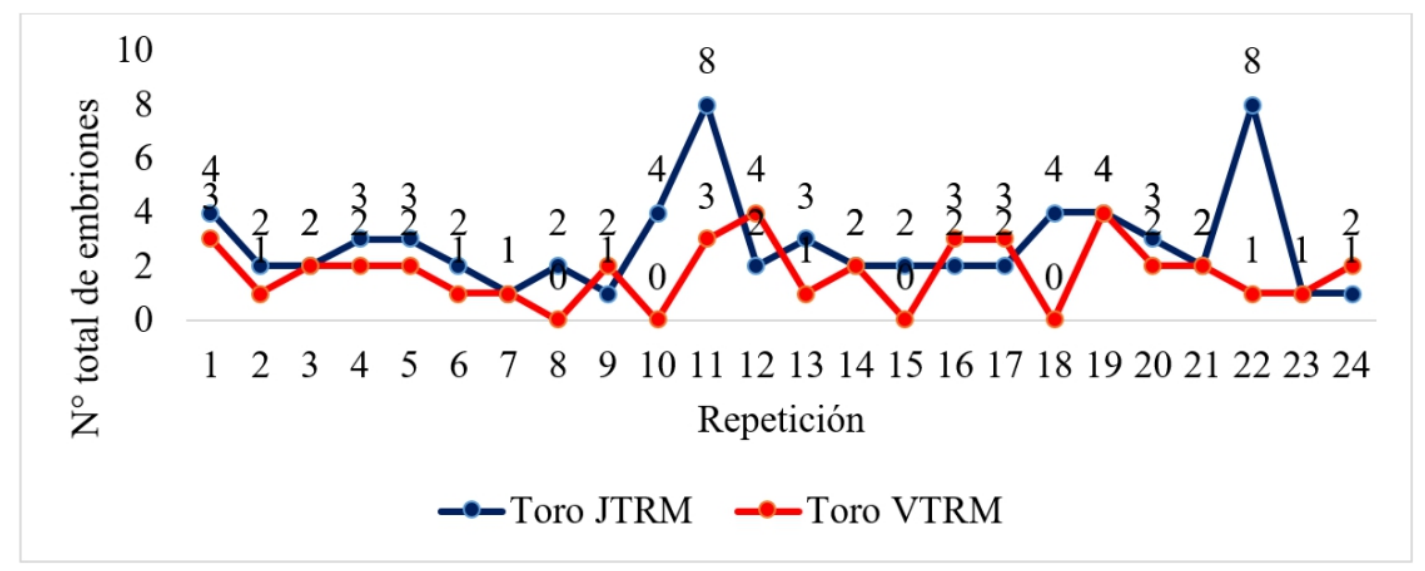

Figura 1. Evaluación de la producción total de embriones 


\section{Producción de blastocistos iniciales}

La figura 2 muestra los números de embriones por repetición que llegaron hasta el estadío de blastocistos iniciales después de la fecundación de
244 óvulos, con promedios porcentuales de $21.31 \%$ a para el toro JTRM y $14.77 \%$ b para el toro VTRM. La diferencia porcentual para esta variable es $6.54 \%$.

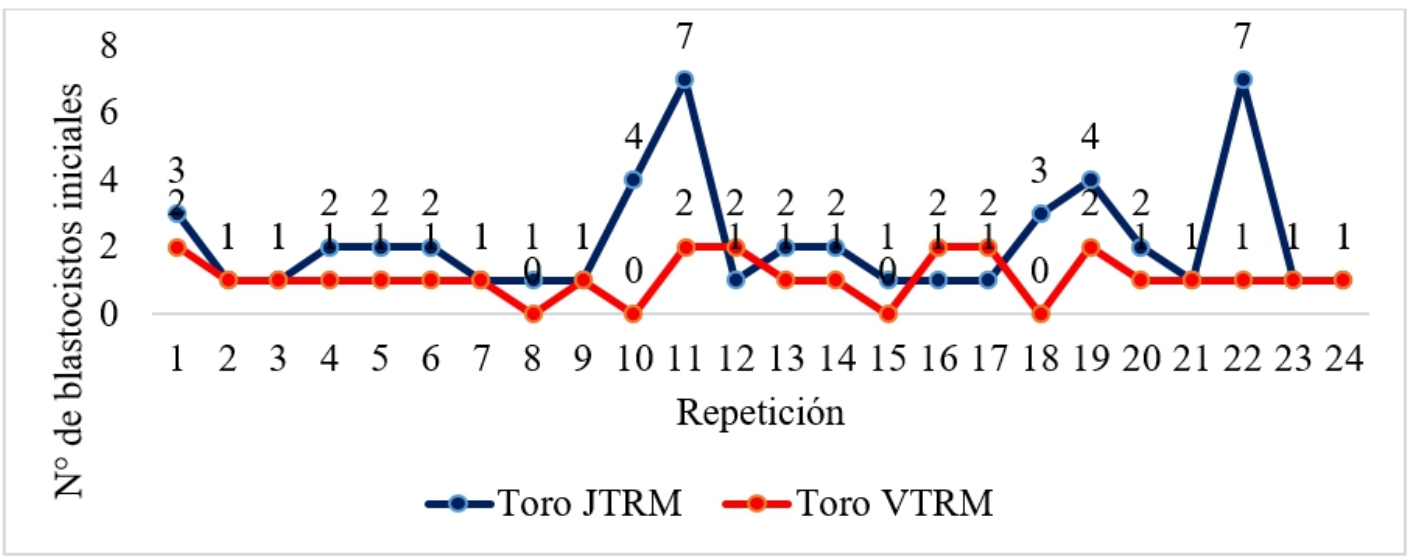

Figura 2. Evaluación de producción de blastocistos iniciales.

\section{Producción de blastocistos}

La media en porcentaje de blastocistos que lograron llegar hasta este estadío es de $5.73 \%$ a y $7.95 \%$ a del total de óvulos fecundados (244) para los toros JTRM y VTRM respectivamente detallada en la figura $3 \mathrm{sin}$ encontrar diferencia estadística para esta variable.

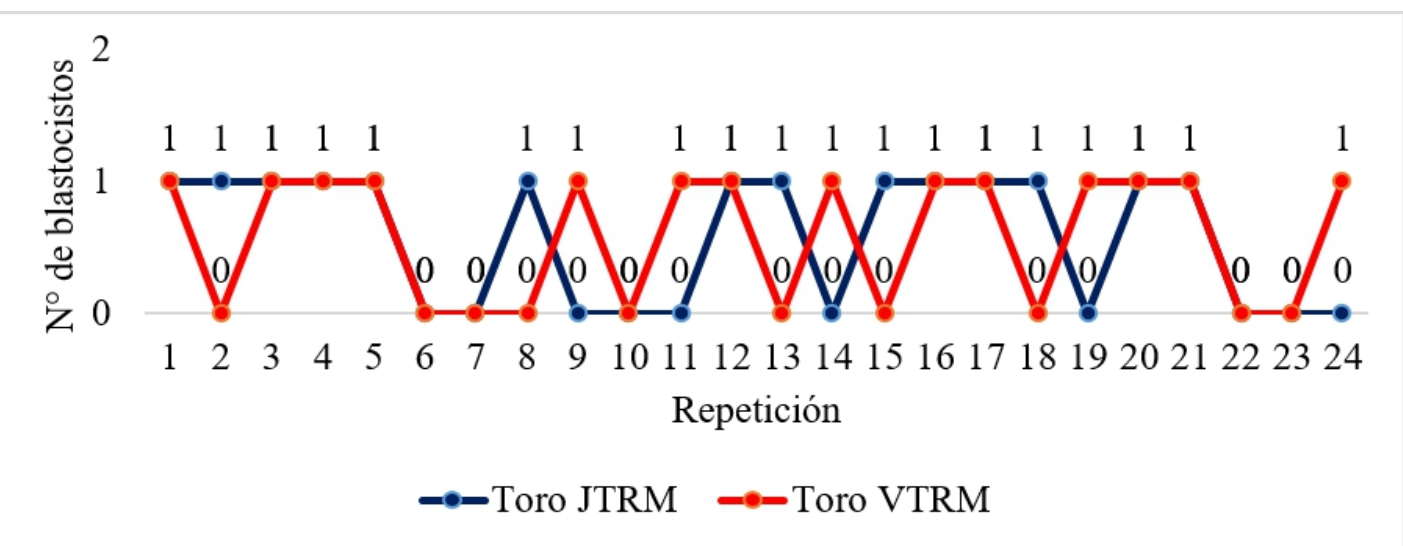

Figura 3. Evaluación de producción de blastocistos.

Producción de blastocistos expandidos

El valor promedio de la producción de blastocistos expandidos que arrojaron las 24 repeticiones fue de $0.82 \%$ a para el toro JTRM, mientras que para el toro
VTRM la media fue de $1.13 \%$ a, del total de óvulos que llegaron a ser fecundados, encontrando una diferencia porcentual del $0.31 \%$ y esto a la vez no demuestra una diferencia significativa.

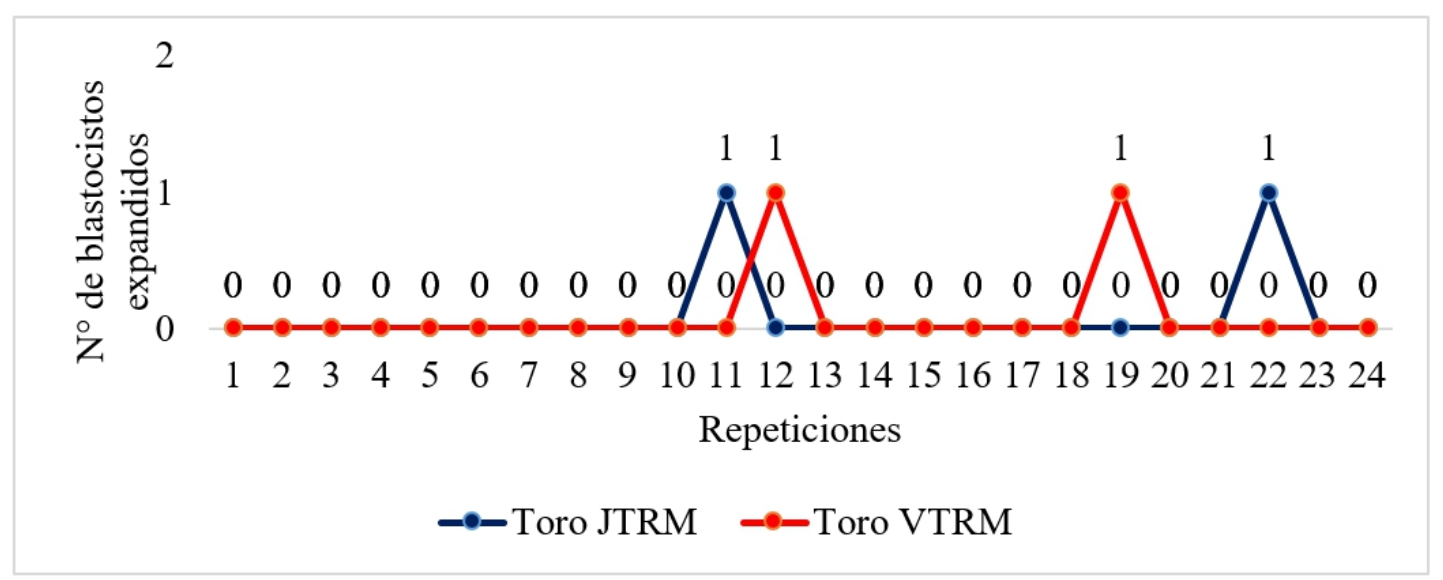

Figura 4. Evaluación de producción de blastocistos expandidos 
Tabla 2: Resultados de significancia para todas las variables

\begin{tabular}{lccccc}
\hline $\begin{array}{l}\text { Nombre } \\
\text { del toro }\end{array}$ & Bi & Bl & & Bx & TE \\
\hline & & & $\mathbf{\%}$ & & \\
\cline { 2 - 6 } JTRM & $21.31^{\mathrm{a}}$ & 5.73 & & 0.82 & $27.86^{\mathrm{a}}$ \\
VTRM & $14.77^{\mathrm{b}}$ & 7.95 & & 1.13 & $23.86^{\mathrm{b}}$ \\
\hline
\end{tabular}

Bi: blastocistos iniciales. Bl: blastocistos. Bx: blastocistos expandidos. TE: total de embriones

a,b letras diferentes en la misma columna indican diferencias estadísticamente significativa

\section{DISCUSIÓN}

El mejor indicador de eficiencia en producción de embriones in vitro es la cantidad y calidad de blastocistos producidos al día 7 post fecundación (Lonergan et al., 2006).

Según Rizos et al., (2002) solo el 30 a 40\% de los ovocitos iniciales tienen la capacidad para llegar al estado de blastocistos. En esta investigación los porcentajes de producción embrionaria estuvieron por debajo de los promedios óptimos de producción (27.86\% el toro JTRM y $23.86 \%$ el toro VTRM). Bonilla et al., (2018) también encontraron resultados de producción de embriones in vitro cercanos a los encontrados en esta investigación donde sus resultados fuero de $22.2 \%$ con semen de toros Bos taurus. Estos resultados también los presentan Medina et al., (2002) donde los valores de producción embrionaria fueron de $26,3 \%$ y $28,8 \%$ de dos toros diferentes, pero se contradicen a los resultados de la investigación de Palma et al., (2007) ellos encuentran un porcentaje de producción de blastocistos del 33,6\%, Presicce et al., (2011) de $39.8 \%$ y Dayan (2001) 36.2\%. Sin embargo, Urrego et al., (2008) muestra una tasa inferior de embriones que los resultados encontrados en esta investigación que van desde un 18 a $20 \%$.

\section{CONCLUSIONES}

La mayor producción total de embriones in vitro en los estadíos de blastocistos iniciales (Bi), blastocistos (Bl) y blastocistos expandidos (Bx) se obtuvo con el uso del semen del ejemplar JTRM $27.86 \%$, frente al uso del semen del ejemplar VTRM $23.86 \%$. Finalmente la variación epigenética estadísticamente significativa en la producción de embriones in vitro difiere en $4 \%$.

\section{REFERENCIAS BIBLIOGRÁFICAS}

Barth, A. (1992). The relations $h i p$
betweensperm abnormalities a $n d$
fertility. In: Proce ding s $14 \mathrm{th}$
Technnical Conference on Artificial

Insemination and Reproduction, 47-63

Bonilla, L.; Mejía, A.; Gómez, R.; Torres, M . ; Uribe, F. (2018). Viabilidady tasa de preñez de embriones producidos in vitro a partirdesemen $s \quad e \quad x \quad a \quad d \quad o$ comparadoconsemen convencional en Bostaurus y Bos indicus. Rev Inv Vet Perú, (4) 1377- 1385

Casas, A.; Cianzio, D.; Rivera, A.; Cantisani, L.; Añeses, L. (2001). Estimación de la e d a d del ganado vacuno por sus incisivos. Estación Experimental Agrícola, $1-6$.

Correa, J.; Pace, M.; Zavos, P. (1997). Relationships among frozenthawedsperm characteristics assessed via the routine semen analysis, sperm functional tests and fertility of bulls in an artificial ins e mination program, Theriogenology (48) 721-731.

Dayan, A. (2001). Fatoresque interferem na produçãode embriões bovinos mediante aspiração folicular e fecundação in vitro. Tese magistral.

De Loos, F.; Van Maurik, P.; Van B e n e d e n , T.; Kruip, A.M. (1992). Structural aspects of bovine oocyte maturation in vitro. Mol. Reprod. Dey, (31) 208214.

Gonzales, A.; Díaz, A. y Díaz- Anzaldúa, A. (2008).La epigenética y los estudiosen gemelosenel campo dela psiquiatría. Salud Mental,(31) 230.

Hirao, K. (1975). A multiple regression analysis on six measurements of bovine semen characteristics for 
fertility. Int. J. Fertil, (20) 204-208.

Holt, W.V.; Brienj, O.; Abaigar, T. ( ( $\left.\begin{array}{lllll}2 & 0 & 0 & 7\end{array}\right)$. Applications and interpretation of computer-assisted sperm analyses and spermsorting methods in assisted breedingand comparative research. Reproduction, Fertility and Development, (19) 709-718.

Januskauskas, A.; Johannisson, A.; S o d e r q u i s t Y.L.; Rodríguez- Martínez, H. (2000). Assessment of sperm characteristics post-thawand response to calcium ionophore in relation to fertility in Swedishdairy AI bull. Theriogenology, (53) $859-875$.

Kjaestad,H.; Ropstad, E.; Andersen B e $r$ g , K. (1993). Evaluation of spermatological parameters used to predict the fertility of frozen bull semen. Acta Vet. S c and, (34) 299-303.

Lonergan, P.; Fair, T.; Corcoran, D.;

Evans. A. (2006). Effect of culture environment on gene expression and developmental characteristics in IVFderived e mbryos. Theriogenology, (65) 137-152.

Medina, M.; Cattaneo, L.; Caballero, J.; Cerrate, H.; Panarace, M.; Ferré, L.; Dalla, M. (2002). Semen sexado $y$ congelado en Argentina. Resultados de su utilización en programasde $\quad i n s$ e mi nación artificial, transferencia embrionaria $y \quad$ fertilizaciónin $v \quad i \quad t \quad r \quad o$. Taurus, (13) 4-8.

Palmer, E.; Bezard, J.; Magistrini, M.; Duchamp, G. (1991). in vitro in the horse. A retrospective study. J. Reprod. Fertil, (44) 375-384.

Perez, L.; Valcarcel, A.; De Las Heras, M.; B aldassarre, H . ( 1997 ). Comparative study of four techniques for evaluation of sperm quality in ovine and bovine frozenthawed samples, Reprod. Domest. Anim (32) 157-160.

Presicce, G.A.; Jiang, S.; Sinkin, M.; Yang, X. (2011). Oocyte quality and embry development in prepubertal calves. Biology of Reproduction, (52) 127.

Quintero, A.; Mayorga, J.; Cardona,

$\mathrm{W}$

(2017). El análisis seminal como herramienta para predecir el potencial reproductivo en toros. Journal of Veterinary Andrology, ISSN 2542-3045, (2) 31.

Rizos, D.; Lonergan. P.; Ward, F.; Duffy, P.; B o l a n d, M. P. ( ( $\left.\begin{array}{llll}2 & 0 & 0 & 2\end{array}\right)$. Consequences of bovine oocyte maturation, fertilization or early embryo development in vitro versusinvivo: implications for blastocyst yield and blastocyst quality. Mol Reprod Dev, (61) 234248.

Sato, E.; Matsuo, M.; Miyamoto, H. (1990). Meiotic maturation ofbovine o o c y tes in vitro: improvement of $m$ e $\quad i \quad r \quad t \quad i \quad c$ competence by dibutyryl $\quad c \quad y \quad c \quad l \quad i \quad c$ adenosine 3', 5' monophosphate. J. Anin. Sci, (68) 1872-1887.

Shamsuddin, M.; Larsson, B. (1993). in vitro development of bovine embryosafter fertilization using semen from different donors. Reprod. Domest. Anim, (28) 77-84.

Shi, D.; Lu, K.; Gordon, I. (1990). Effects of bulls on fertilization of bovine oocytes and their subsequent development in vitro. Theriogenology, (33) 324.

Urrego, R.; Tarazona, A.; Olivera, M.; $\mathrm{C}$ a m a r g o, O. $\quad\left(\begin{array}{llll}2 & 0 & 0 & 8\end{array}\right)$. Simplificación de la fertilización de ovocitos durante la producción in vitro de embriones bovinos. Rev. Col. Cien. Pec., (3) 398-405.

Zhang, B.; Larsson, B.; Lundeheim, N . ; R o d ríg u e z - M a r tín e z, H . (1998). $S \quad p \quad$ e r $\quad r \quad m$ characteristics and zona pellucida binding in relation to field fertility of frozen-thawed semen from dairy AI bulls. Int. J. Androl, (21) 207-216. 